\title{
Low-scale quintessential inflation
}

\author{
Massimo Giovannini* \\ Theoretical Physics Division, CERN, CH-1211, Switzerland
}

(Received 31 January 2003; published 20 June 2003)

\begin{abstract}
In the quintessential inflationary model, the same master field that drives inflation becomes, later on, the dynamical source of the (present) accelerated expansion. Quintessential inflationary models require a curvature scale at the end of inflation around $10^{-6} M_{\mathrm{P}}$ in order to explain the large scale fluctuations observed in the microwave sky. If the curvature scale at the end of inflation is much smaller than $10^{-6} M_{\mathrm{P}}$, the large scale adiabatic mode may be produced thanks to the relaxation of a scalar degree of freedom, which will be generically denoted, according to the recent terminology, as the curvaton field. The production of the adiabatic mode is analyzed in detail in the case of the minimal quintessential inflationary model originally proposed by Peebles and Vilenkin.
\end{abstract}

DOI: 10.1103/PhysRevD.67.123512

PACS number(s): 98.80.Cq, 98.70.Vc

\section{INTRODUCTION}

Following the discovery $[1,2]$ that distant supernovas are fainter than inferred from local samples, models of scalar fields that are able to develop a negative pressure at the present time have been proposed. These scalar fields only interact gravitationally and they have been generically named quintessence [3-8].

In recent years, an interesting class of quintessence models has been proposed by Peebles and Vilenkin [9] (see also $[10]) .^{1}$ The peculiar feature of quintessential inflation is that the master field that drove inflation becomes again dominant, at the present time. Hence, in the context of quintessential inflation, the inflaton and the quintessence field are identified in a single scalar degree of freedom, driving inflation in the past and acting as quintessence today. A simple example of this dynamics is represented by "dual" potentials [9] going as a power during inflation and as an inverse power after [4] inflation. Owing to this dual form of the potential, the dynamics of the background will experience, right after inflation, a pretty long phase in which the potential term is subleading with respect to the kinetic term. In quintessential inflation the reheating can be entirely gravitational, since the energy density in the light quanta produced at the end of inflation decreases slower than the (kinetic-energydominated) background geometry $[9,12]$.

Quintessential inflationary models are consistent with observations provided the curvature scale at the end of inflation is not too low and $O \sim\left(10^{-6} M_{\mathrm{P}}\right)$. In the present paper a complementary possibility will be analyzed. Consider the situation where $H_{\mathrm{e}}$, the curvature scale at the end of inflation, is indeed much smaller than $10^{-6} M_{\mathrm{P}}$. Along this line we suppose, for simplicity, that during inflation there is degree of freedom $\psi$ which is not coupled with the inflaton field and remains constant, thanks to its potential, during the later stages of inflation. The (potential) energy density of $\psi$

\footnotetext{
*Electronic address: massimo.giovannini@cern.ch

${ }^{1}$ The early "history" of the identification between the inflaton and the quitessence field can be found in [11]. In addition to [3,4], relevant references are, in this context, [12] and [13].
}

is subleading with respect to the energy density of $\varphi$. At the end of inflation, during the kinetic phase, the large scale fluctuations of $\psi$ will be converted into adiabatic fluctuations. This possibility has recently been studied by many authors $[14-20]$ in different contexts, and it was originally invoked in [21]. As discussed in [22], even the simplest chaotic inflationary models develop new constraints when combined with the curvaton idea. The purpose of the present paper is to analyze low-scale quintessential inflation in a specific setup, which is the one originally suggested by Peebles and Vilenkin in [9]. In this model the late-time behavior of the quintessential evolution does not show the tracking behavior of the inflaton and matter mass densities, as argued in [7].

Particular attention will be given to the evolution of the fluctuations. This is quite essential since, by lowering the curvature scale at which inflation ends, we have to make sure that the isocurvature mode of $\psi$ is efficiently turned into an adiabatic one. While in ordinary inflationary models (such as those analyzed, for instance, in [22]) the radiation-dominated phase starts at the end of inflation, in quintessential inflation the evolution may be very different and the onset of the radiation-dominated phase is delayed. One of the purposes of the present paper is indeed to generalize the analysis of the curvaton evolution to backgrounds where inflation is not immediately followed by a radiation-dominated phase. The curvature perturbation will be followed through all the stages of the model and its final value computed. This analysis will be performed both analytically and numerically.

The plan of the present paper is the following. In Sec. II the constraints on the post-inflationary evolution will be derived in the specific case where the inflaton and quintessence field are identified. In Sec. III the constraints pertaining to the quintessential evolution will be scrutinized. Section IV contains the basic ingredients for the evolution of the fluctuations. Sections V and VI deal with the conversion of the initial isocurvature mode into an adiabatic one. In Sec. V the initial and the kinetic stages will be scrutinized, while Sec. VI is more oriented towards the phase where $\psi$ dominates and eventually decays. In Sec. VII some concluding remarks will be presented. 


\section{FROM INFLATION TO QUINTESSENCE}

Consider the minimal realization of a low-scale quintessential inflationary model extending the original proposal of [9]:

$$
\begin{gathered}
M_{\mathrm{P}}^{2} H^{2}=\left[\frac{\dot{\varphi}^{2}}{2}+\frac{\dot{\psi}^{2}}{2}+V(\varphi)+W(\psi)\right], \\
M_{\mathrm{P}}^{2}\left(H^{2}+\dot{H}\right)=\left[-\dot{\varphi}^{2}-\dot{\psi}^{2}+V(\varphi)+W(\psi)\right], \\
\ddot{\varphi}+3 H \dot{\varphi}+\frac{\partial V}{\partial \varphi}=0, \\
\ddot{\psi}+3 H \dot{\psi}+\frac{\partial W}{\partial \psi}=0 .
\end{gathered}
$$

The potential of $\varphi$ can be chosen to be a typical power law during inflation and an inverse power during the quintessential regime:

$$
\begin{gathered}
V(\varphi)=\lambda\left(\varphi^{4}+M^{4}\right), \quad \varphi<0, \\
V(\varphi)=\frac{\lambda M^{8}}{\varphi^{4}+M^{4}}, \quad \varphi \geqslant 0,
\end{gathered}
$$

where $\lambda$ is the inflaton self-coupling and $M$ is the typical scale of quintessential evolution.

The field $\psi$ is subleading during inflation and it is characterized by a potential, which we will take, for simplicity, to be quadratic, i.e.

$$
W(\psi)=\frac{m^{2}}{2} \psi^{2} .
$$

This setup can be generalized to the case where the field $\psi$ is replaced by an arbitrary number of scalar degrees of freedom $\psi_{i}$.

Inflation ends when $V(\varphi) \sim \lambda M_{\mathrm{P}}^{4}$ at a curvature scale $H_{\mathrm{e}}$

$$
\sqrt{\lambda} \simeq \frac{H_{\mathrm{e}}}{M_{\mathrm{P}}} .
$$

In ordinary inflationary models, the large scale inhomogeneities determining the Cosmic microwave background (CMB) anisotropies come from the Gaussian fluctuations of the inflaton $\varphi$, and, consequently, $\lambda \sim 10^{-13}$ [9]. In the present investigation a complementary possibility will be discussed, namely the case when

$$
H_{\mathrm{e}} \ll 10^{-6} M_{\mathrm{P}}
$$

In this case the fluctuations of $\varphi$ are too small to be interesting for $\mathrm{CMB}$ physics. However, this conclusion can be evaded by taking into account the fluctuations of the field $\psi$. Qualitatively the picture is the following. Right after inflation, the fluctuations of the geometry will be determined by the fluctuations both of $\varphi$ and $\psi$. Because of Eq. (2.8), the metric fluctuations generated by $\varphi$ will vanish at $t_{\mathrm{e}}$. In other words, the initial conditions for the system at $t_{\mathrm{e}}$ will be of isocurvature type. Later on, the isocurvature mode can be converted into an adiabatic one. This is the idea explored, for instance, in $[14,15]$. In the usual picture of curvaton evolution, right after inflation, radiation takes place immediately. In the case of quintessential inflation, on the contrary, the onset of the radiation-dominated epoch may be delayed. Hence, the analysis of the evolution of the fluctuations must be repeated, with particular attention to the different features of the model.

During inflation, the field $\psi$ is subdominant with respect to the inflaton energy density,

$$
W(\psi) \ll V(\varphi) .
$$

The field $\psi$ remains nearly constant during the later stages of inflation, i.e. $\psi \sim \psi_{\mathrm{e}}$. At the end of inflation, Eq. (2.9) implies that

$$
\left(\frac{m}{M_{\mathrm{P}}}\right) \ll \sqrt{2}\left(\frac{H_{\mathrm{e}}}{\psi_{\mathrm{e}}}\right) .
$$

After the end of inflation, because of the inverse powerlaw form of the potential, the field $\varphi$ is mainly driven by its kinetic energy and the approximate solution of the background geometry will be of the type

$$
\varphi=\sqrt{2} M_{\mathrm{P}} \ln \left(\frac{a}{a_{\mathrm{e}}}\right),
$$

with $a(t) \sim t^{1 / 3}$. In this phase the potential, $V(\varphi)$ $\sim \lambda\left(M / M_{\mathrm{P}}\right)^{4} \ln ^{-4}\left(a / a_{\mathrm{e}}\right)$, is subleading since $\lambda \ll 10^{-13}$ and $M \ll M_{\mathrm{P}}$. More specifically, phenomenological considerations related to the present dominance of $\varphi$ (see Sec. III) suggest that $M \ll 10^{-9} M_{\mathrm{P}}$.

While the background is dominated by $\dot{\varphi}^{2}$, the field $\psi$ slightly decreases with a rate given by $W_{, \psi}$. From Eq. (2.4), the field $\psi$ slowly rolls toward the minimum of its potential in a kinetic-energy-dominated environment and its approximate equation obeys

$$
\dot{\psi} \simeq-\frac{1}{6 H} \frac{\partial W}{\partial \psi},
$$

where the factor 1/6 comes from dropping consistently the terms of Eq. (2.4) containing more than one derivative of the potential. This type of approximation has been exploited in [16], but in the case of a slow-roll occurring during the radiation epoch.

Using Eq. (2.12), and recalling that $a(t) \sim t^{1 / 3}$, it can be checked that the evolution of $\psi$ slightly deviates from a constant value. For instance, in the case of a quadratic potential the solution of Eq. (2.12) can be written as

$$
\psi(t) \simeq \psi_{\mathrm{e}}\left[1-\frac{m^{2}}{4}\left(t^{2}-t_{\mathrm{e}}^{2}\right)\right] .
$$

Equation (2.12) is useful in the case when the potential is more complicated than the quadratic ansatz of Eq. (2.6) where Eq. (2.4) can be solved exactly. In fact, during the 
kinetic regime the curvature scale decreases, and when $H$ $\sim H_{\text {osc }} \sim m$ the field $\psi$, still subdominant, will start oscillating. The case of massive potential allows analytical solutions of Eq. (2.4) during the kinetic phase for $\psi$ :

$$
\psi=\psi_{\mathrm{e}} \frac{\sqrt{t}}{a^{3 / 2} J_{0}\left(m t_{\mathrm{e}}\right)} J_{0}(m t)
$$

which leads to Eq. (2.12) in the limit $m t \ll 1$ and $m t_{\mathrm{e}} \ll 1$. Since $\psi$ decays as $a^{-3 / 2}$ during the oscillating regime, it will become dominant with respect to the kinetic energy of $\varphi$ at a scale

$$
H_{\mathrm{d}} \sim 5 \quad m\left(\frac{\psi_{\mathrm{e}}}{M_{\mathrm{P}}}\right)^{2}
$$

The field $\psi$ eventually decays at a curvature scale

$$
H_{\mathrm{r}} \sim \frac{m^{3}}{M_{\mathrm{P}}^{2}} .
$$

At $H_{\mathrm{m}}$ the potential of $\varphi$ is still subdominant with respect to the kinetic energy. In fact

$$
\begin{aligned}
V\left(\varphi_{\mathrm{m}}\right) & \sim \lambda M^{4}\left(\frac{M}{M_{\mathrm{P}}}\right)^{4} \ln ^{-4}\left(\frac{H_{\mathrm{e}}}{m}\right) \\
\ll & \frac{\dot{\varphi}_{\mathrm{m}}^{2}}{2} \simeq \frac{1}{9}\left(\frac{M_{\mathrm{P}}^{2}}{m^{2}}\right),
\end{aligned}
$$

where the last equality comes from the kinetic term of $\varphi$ at $t_{\mathrm{m}}$, evaluated on the basis of Eq. (2.11). An example of the numerical integration is reported in Fig. 1. Furthermore, the analytical evolution of $\psi$, as reported in Eq. (2.14), is in excellent agreement with the numerical results.

Between $H_{\mathrm{d}}$ and $H_{\mathrm{r}}$,

$$
\left(\frac{a_{\mathrm{d}}}{a_{\mathrm{r}}}\right) \simeq\left(\frac{m}{\psi_{\mathrm{e}}}\right)^{2}
$$

the background geometry is effectively dominated by the oscillations of $\psi$, i.e. $a(t) \sim(m t)^{2 / 3}$.

It is interesting to combine in the physical picture the constraints and the requirements introduced so far. According to Eqs. (2.15) and (2.16) the quintessence field may become subdominant either prior to or after the decay of $\psi$. In Fig. 2, with the (diagonal) dashed line, the condition coming from the interplay between the decay of $\psi$ and the dominance of $\varphi$ is illustrated for the specific case $H_{\mathrm{e}} \sim 10^{-9} M_{\mathrm{P}}$. Above the dashed line, $\psi$ decays during the kinetic phase. Below the dashed line, the decay occurs when $\psi$ already dominates. The requirement of Eq. (2.10) also imposes a constraint on Fig. 2, implying that $\psi_{\mathrm{e}}$ and the mass should lie below the full fine in the right-hand corner. Consider now the situation where the fluctuations of $\psi$ are amplified, during inflation, with a scale-invariant spectrum. Therefore, denoting by $\chi_{\psi}$ the fluctuation of $\psi$, we do know that the power spectrum will be $\delta_{\chi_{\psi}} \sim H_{\mathrm{e}} / 2 \pi$. If
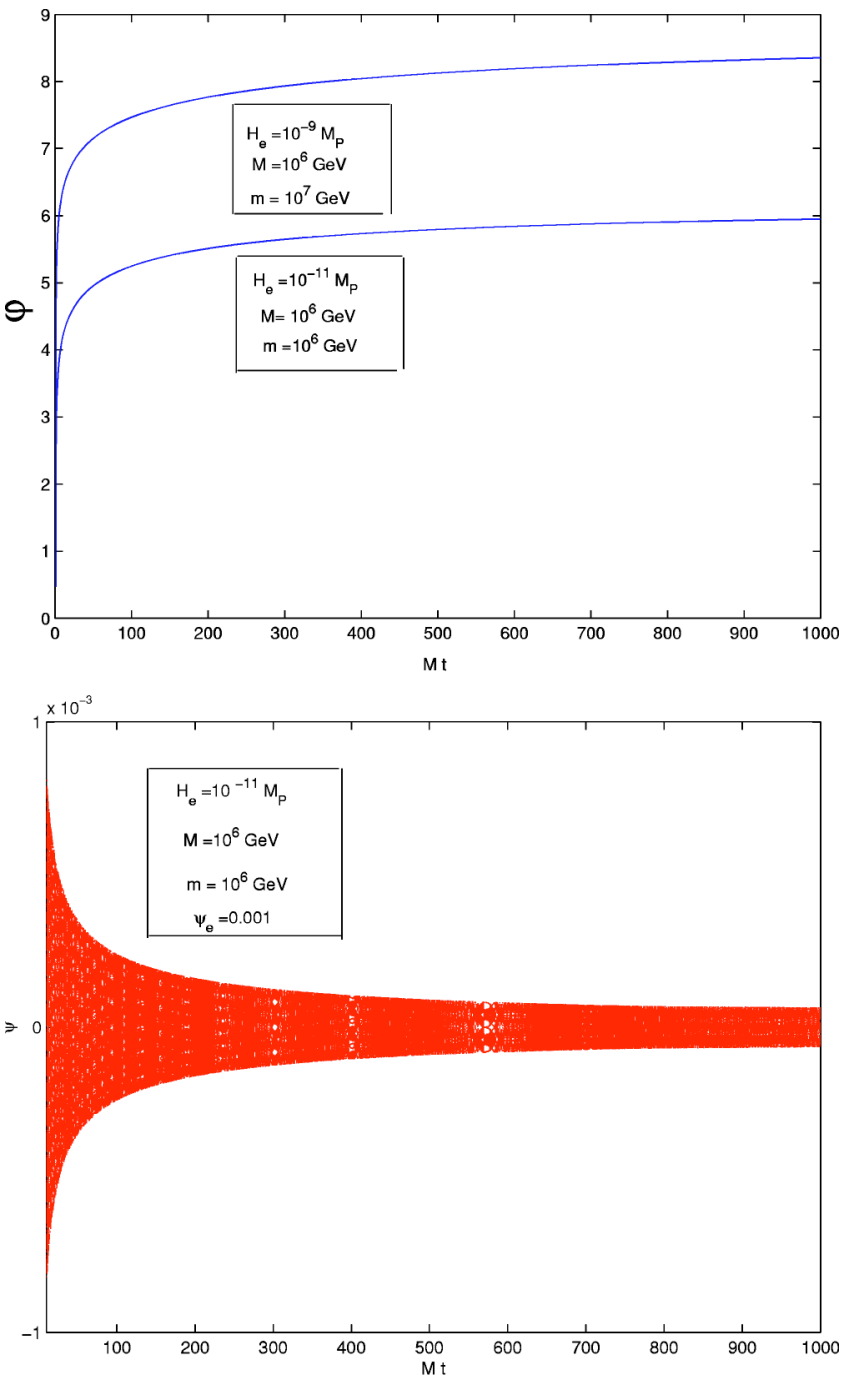

FIG. 1. The result of the numerical integration of the background is illustrated for different values of the parameters (indicated above each curve).

$$
\frac{H_{\mathrm{e}}}{M_{\mathrm{P}}}<2 \pi \frac{\psi_{\mathrm{e}}}{M_{\mathrm{P}}}
$$

then the fluctuations of $\psi$ will be predominantly Gaussian. In the opposite case they will have some non-negligible nonGaussian component. In order to have a nearly scaleinvariant spectrum for $\chi_{\psi}, m$ should always be smaller than the curvature scale $H_{\mathrm{e}}$. More precisely, looking at the evolution equation for $\chi_{\psi}$ (in conformal time) we are led to require

$$
m<\sqrt{2} H_{\mathrm{e}} .
$$

Equation (2.20) is illustrated in Fig. 2 with the dotted horizontal line. Finally, since the decay of $\psi$ should occur prior to BBN, an absolute lower bound on $m$, i.e. $m>10 \mathrm{TeV}$, should be imposed. Therefore, already from these considerations it is possible to say that the dashed area in Fig. 2 is allowed. Thus, the quintessence field has to become sub- 


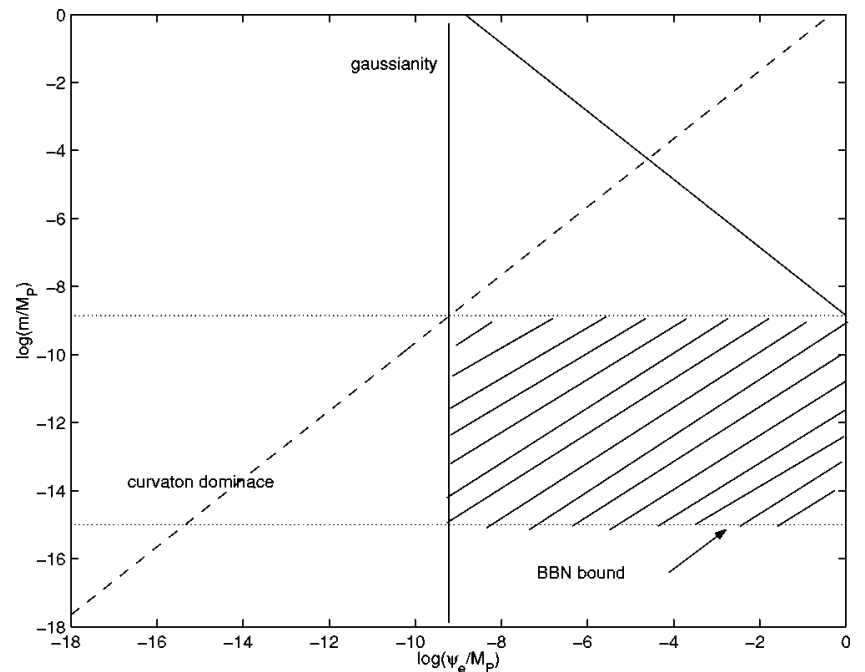

FIG. 2. The quantitative constraints pertaining to the combined analysis of the evolution of $\psi$ and $\varphi$ during the kinetic phase are illustrated. With the dashed line the constraint coming from the dominance of $\psi$ is reported. The dot-dashed lines (from top to bottom) represent, respectively, the bound (2.20) and the big bang nuclearynthesis (BBN) bound. The full (vertical) line refers to Eq. (2.19). The shaded region defines the allowed portion of the parameter space of the model.

dominant before the decay of $\psi$ if we want $\chi_{\psi}$ to be Gaussian with a nearly scale-invariant spectrum.

\section{QUINTESSENTIAL EVOLUTION}

In order to satisfy the physical constraints for the consistency of the scenario, it is reasonable to require that the decay of $\psi$ occur when $\varphi$ is already subdominant. If this is the situation, $H_{\mathrm{d}}>H_{\mathrm{r}}$ and, consequently,

$$
\psi_{\mathrm{e}} \gtrsim \frac{1}{\sqrt{5}} m
$$

It is important, for the purposes of the present investigation, to analyze in some detail, the evolution of $\varphi$ around the curvature scales $H_{\mathrm{d}}$ and $H_{\mathrm{r}}$.

After $H_{\mathrm{d}}$, the evolution of $\varphi$ results from the interplay between the smallness of its potential term $V(\varphi)$ and the coherent oscillations of $\psi$, which tend to make the average expansion matter-dominated. The effect of the potential is, however, to introduce a growing mode in the evolution of $\varphi$. This growing mode can be estimated by solving Eq. (2.3) with the inverse power-law potential of Eq. (2.5). The solution is

$$
\varphi_{\mathrm{g}}(t) \simeq 3^{1 / 3} \quad \lambda^{1 / 6} M^{4 / 3} t^{1 / 3}, \quad t_{\mathrm{d}}<t<t_{\mathrm{r}} .
$$

For $t>t_{\mathrm{r}}$, when the Universe is effectively radiationdominated, a similar solution holds:

$$
\varphi_{\mathrm{g}}(t) \simeq\left(\frac{72}{5}\right)^{1 / 6} \quad \lambda^{1 / 6} M^{4 / 3} t^{1 / 3}, \quad t>t_{\mathrm{r}}
$$

The effective evolution of $\varphi$ depends upon the balance of the growing mode with the decaying modes obtained from Eq. (2.3) when the potential is neglected. During the regime where $\psi$ dominates and in the subsequent radiationdominated regime, the evolution of $\varphi$ is given, respectively, by

$$
\begin{gathered}
\varphi_{\mathrm{d}}(t) \simeq \frac{\sqrt{2} M_{\mathrm{P}}}{3}\left\{\left[\ln \left(\frac{t_{\mathrm{d}}}{t_{\mathrm{e}}}\right)+1\right]-\frac{t_{\mathrm{d}}}{t}\right\}, \\
t_{\mathrm{d}}<t<t_{\mathrm{r}}, \\
\varphi_{\mathrm{r}}(t) \simeq \frac{\sqrt{2} M_{\mathrm{P}}}{3}\left\{\left[\ln \left(\frac{t_{\mathrm{d}}}{t_{\mathrm{e}}}\right)+1+\frac{t_{\mathrm{d}}}{t_{\mathrm{r}}}\right]-2 \frac{t_{\mathrm{d}}}{t_{\mathrm{r}}} \sqrt{\frac{t_{\mathrm{r}}}{t}}\right\}, \\
t>t_{\mathrm{r}} .
\end{gathered}
$$

Equations (3.4) and(3.5) are continuous in $t_{\mathrm{r}}$. Furthermore Eqs. (2.11) and (3.4) are continuous in $t_{\mathrm{d}}$. As obtained in [9] the growing solution never comes to dominate against the decreasing mode. If

$$
\lambda^{-1 / 2} \ln ^{3}\left(\frac{t_{\mathrm{d}}}{t_{\mathrm{e}}}\right)\left(\frac{M_{\mathrm{P}}}{M}\right)^{4}>\left(\frac{H_{0}}{M_{\mathrm{P}}}\right)^{-1},
$$

the growing mode becomes dominant only after the present expansion time $t_{0} \sim H_{0}^{-1}$. We will see that this condition is always verified because of the smallness of $\lambda$.

After $t_{\mathrm{r}}$ the quintessence field is nearly constant and its potential energy is given by

$$
V\left(\varphi_{\mathrm{r}}\right) \simeq \lambda \frac{M^{8}}{M_{\mathrm{P}}^{4}}\left[\ln \left(\frac{t_{\mathrm{d}}}{t_{\mathrm{e}}}\right)\right]^{-4}
$$

The condition that the potential energy in the quintessence field is comparable with the present energy density,

$$
V\left(\varphi_{\mathrm{r}}\right) \simeq H_{0}^{2} M_{\mathrm{P}}^{2}
$$

fixes a relation among $M$ and $\lambda$

$$
\begin{aligned}
\frac{M}{M_{\mathrm{P}}} \simeq & \left(\frac{9}{2}\right)^{-1 / 4}\left(\frac{H_{0}}{M_{\mathrm{P}}}\right)^{1 / 4} \\
& \times \lambda^{-1 / 8}\left\{\ln \left[\frac{\sqrt{\lambda}}{5}\left(\frac{M_{\mathrm{P}}}{m}\right)\left(\frac{M_{\mathrm{P}}}{\psi_{\mathrm{e}}}\right)^{2}\right]\right\}^{1 / 2} .
\end{aligned}
$$

Note that, for the typical parameters discussed so far, $M$ is always greater than $10^{5} \mathrm{GeV}$.

\section{LARGE SCALE FLUCTUATIONS}

Having discussed the main features of the postinflationary evolution, it is now mandatory to understand the behavior of the fluctuations. Available calculations on the curvaton dynamics in different models [14-20] always deal with a post-inflationary phase, which is dominated by radiation. Here, as discussed in the previous sections, the scenario is different. Right after inflation the background evolution is 
not determined by radiation but by the dynamics of $\varphi$, whose potential is now very steep. The fluctuation of the metric induced by the fluctuations of $\varphi$ will be, at the onset of the post-inflationary epoch, very small since $H_{\mathrm{e}}<10^{-6} M_{\mathrm{P}}$. Hence, had we to classify the metric fluctuations at $t_{\mathrm{e}}$, we would say that their modes are of the isocurvature type. However, as time goes by, the curvature fluctuations, initially negligible at $t_{\mathrm{e}}$, will be driven to a constant value so that, much later, the initial isocurvature mode turns into an adiabatic one.

In order to describe this picture quantitatively, we are led to consider the coupled system formed by the fluctuations of the metric and by the fluctuations of $\varphi$ and $\psi$. The fluctuations will then be discussed in the longitudinal gauge, where it is particularly simple to relate the gauge-dependent quantities to gauge-invariant observables [23,24].
In the longitudinal gauge, the nonvanishing entries of the perturbed metric are

$$
\delta g_{00}=2 \phi, \quad \delta g_{i j}=-2 a^{2} \phi
$$

where we used the fact that the fluctuations of the energymomentum tensor are free of shear. For the fluctuations of $\varphi$ and $\psi$, in the longitudinal gauge the following notation will also be adopted:

$$
\begin{gathered}
\varphi \rightarrow \varphi+\delta \varphi, \quad \delta \varphi=\chi_{\varphi}, \\
\psi \rightarrow \psi+\delta \psi, \quad \delta \psi=\chi_{\psi} .
\end{gathered}
$$

With these notations the evolution equations of the fluctuations can be written as

$$
\begin{gathered}
\ddot{\phi}+4 H \dot{\phi}+\left(2 \dot{H}+3 H^{2}\right) \phi=-\frac{3}{2 M_{\mathrm{P}}^{2}}\left[\left(\dot{\varphi}^{2}+\dot{\psi}\right)^{2} \phi-\left(\dot{\psi} \dot{\chi}_{\psi}+\dot{\varphi} \dot{\chi}_{\varphi}\right)+\frac{\partial V}{\partial \varphi} \chi_{\varphi}+\frac{\partial W}{\partial \psi} \chi_{\psi}\right], \\
3 H(H \phi+\dot{\phi})-\frac{1}{a^{2}} \nabla^{2} \phi=-\frac{3}{2 M_{\mathrm{P}}^{2}}\left[-\left(\dot{\varphi}^{2}+\dot{\psi}\right)^{2} \phi+\left(\dot{\psi}_{\psi}+\dot{\varphi}_{\varphi}\right)+\frac{\partial V}{\partial \varphi} \chi_{\varphi}+\frac{\partial W}{\partial \psi} \chi_{\psi}\right], \\
\ddot{\chi}_{\varphi}+3 H \dot{\chi}_{\varphi}-\frac{1}{a^{2}} \nabla^{2} \chi_{\varphi}+\frac{\partial^{2} V}{\partial \varphi^{2}} \chi_{\varphi}-4 \dot{\varphi} \dot{\phi}+2 \frac{\partial V}{\partial \varphi} \phi=0, \\
\ddot{\chi}_{\psi}+3 H \dot{\chi}_{\psi}-\frac{1}{a^{2}} \nabla^{2} \chi_{\psi}+\frac{\partial^{2} W}{\partial \psi^{2}} \chi_{\psi}-4 \dot{\psi} \dot{\phi}+2 \frac{\partial W}{\partial \psi} \phi=0,
\end{gathered}
$$

where Eqs. (4.3) and (4.4) come, respectively, from the $(i, j)$ and $(0,0)$ components of the perturbed Einstein equations and Eqs. (4.5) and (4.6) describe the evolution of the inhomogeneities in the inflaton or quintessence field and in the curvaton field. Equations (4.3)-(4.6) are subjected to the momentum constraint

$$
H \phi+\dot{\phi}=\frac{3}{2 M_{\mathrm{P}}^{2}}\left(\dot{\varphi} \chi_{\varphi}+\dot{\psi} \chi_{\psi}\right)
$$

Using Eq. (4.7) together with Eqs. (4.3)-(4.6), it is possible to obtain a nicer form of the perturbation equations [2527,29]:

$$
\begin{gathered}
\ddot{v}_{\varphi}+3 H \dot{v}_{\varphi}-\frac{1}{a^{2}} \nabla^{2} v_{\varphi}+\left[\frac{\partial^{2} V}{\partial \varphi^{2}}-\frac{3}{M_{\mathrm{P}}^{2} a^{3}} \frac{\partial}{\partial t}\left(\frac{a^{3}}{H} \dot{\varphi}^{2}\right)\right] v_{\varphi} \\
-\frac{3}{M_{\mathrm{P}}^{2} a^{3}} \frac{\partial}{\partial t}\left(\frac{a^{3}}{H} \dot{\varphi} \dot{\psi}\right) v_{\psi}=0
\end{gathered}
$$

$$
\begin{aligned}
\ddot{v}_{\psi}+ & 3 H \dot{v}_{\psi}-\frac{1}{a^{2}} \nabla^{2} v_{\psi}+\left[\frac{\partial^{2} W}{\partial \psi^{2}}-\frac{3}{M_{\mathrm{P}}^{2} a^{3}} \frac{\partial}{\partial t}\left(\frac{a^{3}}{H} \dot{\psi}^{2}\right)\right] v_{\psi} \\
& -\frac{3}{M_{\mathrm{P}}^{2} a^{3}} \frac{\partial}{\partial t}\left(\frac{a^{3}}{H} \dot{\varphi} \dot{\psi}\right) v_{\varphi}=0
\end{aligned}
$$

where

$$
\begin{aligned}
& v_{\varphi}=\chi_{\varphi}+\frac{\dot{\varphi}}{H} \phi, \\
& v_{\psi}=\chi_{\psi}+\frac{\dot{\psi}}{H} \phi .
\end{aligned}
$$

Equations (4.8) and (4.9) can be generalized to the case of an arbitrary number of fields $[25,26]$ : in this case the number of equations will clearly match the number of fields but the relative structure of the equations will be the same.

Equations (4.4)-(4.6) or, equivalently, Eqs. (4.8) and (4.9), have to be studied and solved along the different stages of the evolution of the background. In terms of $v_{\varphi}$ and $v_{\psi}$, the spatial curvature perturbation can be written as 


$$
\zeta=-\frac{H}{\dot{\varphi}^{2}+\dot{\psi}^{2}}\left[\dot{\varphi} v_{\varphi}+\dot{\psi} v_{\psi}\right] .
$$

The variable $\zeta$ is related also to $\phi$ by the usual expression

$$
\zeta=\frac{H}{\dot{H}}(H \phi+\dot{\phi})-\phi .
$$

Equation (4.13) can be obtained from Eq. (4.12) (or vice versa) by using the momentum constraint (4.7) expressed in terms of the $v_{\varphi}$ and $v_{\psi}$ given in Eqs. (4.10) and (4.11).

An interesting (complementary) strategy in order to solve the system (4.3)-(4.6) and (4.7) is to write down directly the evolution equation for $\zeta$. Mutiplying Eq. (4.4) by the sound of speed and subtracting it from Eq. (4.3) we obtain, at large scales [28],

$$
\frac{d \zeta}{d t}=-\frac{H}{\dot{\psi}^{2}+\dot{\varphi}^{2}} \delta p_{\text {nad }},
$$

where $\delta p_{\text {nad }}$ is, in our case,

$$
\begin{aligned}
\delta p_{\mathrm{nad}}= & \left(c_{s}^{2}-1\right) \phi(\dot{\varphi}+\dot{\psi})+\left(1-c_{s}^{2}\right)\left(\dot{\varphi} \dot{\chi}_{\varphi}+\dot{\psi} \dot{\chi}_{\psi}\right) \\
& -\left(1+c_{s}^{2}\right)\left(\frac{\partial V}{\partial \varphi} \chi_{\varphi}+\frac{\partial W}{\partial \psi} \chi_{\psi}\right),
\end{aligned}
$$

with

$$
c_{s}^{2}=\frac{\dot{p}}{\dot{\rho}}=1+\frac{2}{3 H\left(\dot{\varphi}^{2}+\dot{\psi}^{2}\right)}\left(\frac{\partial V}{\partial \varphi} \dot{\varphi}+\frac{\partial W}{\partial \psi} \dot{\psi}\right) .
$$

In the first equality of Eq. (4.16) $p$ and $\rho$ are, respectively, the total pressure and energy density of the system written in terms of the two background fields $\varphi$ and $\psi$. Notice that in order to get to Eq. (4.16) the background equations of motion (2.1)-(2.4) have been used.

The initial conditions of the system (4.4)-(4.6) after the end of inflation are dictated by the smallness of $\lambda$. Going to Fourier space and considering only the super-horizon scales, the initial conditions of the system are, for $\lambda \ll 10^{-14}$,

$$
\phi\left(k, t_{\mathrm{e}}\right)=0, \quad \chi_{\varphi}\left(k, t_{\mathrm{e}}\right)=0, \quad \chi_{\psi}\left(k, t_{\mathrm{e}}\right)=\frac{H_{\mathrm{e}}}{2 \pi} .
$$

In terms of $v_{\varphi}$ and $v_{\psi}$, we have at the end of inflation, from Eqs. (4.8) and (4.9), on super-horizon scales,

$$
v_{\varphi}\left(k, t_{\mathrm{e}}\right)=0, \quad v_{\psi}\left(k, t_{\mathrm{e}}\right)=\chi_{\psi}\left(k, t_{\mathrm{e}}\right),
$$

as is made clear by inserting Eqs. (4.17) into Eqs. (4.8) and (4.9). Physically Eqs. (4.17) and (4.18) guarantee the absence of adiabatic modes at $t_{\mathrm{e}}$. This aspect can also be appreciated by looking at the initial conditions for $\zeta$

$$
\zeta\left(k, t_{\mathrm{e}}\right)=0,
$$

as they follow from Eq. (4.12).

\section{EVOLUTION DURING THE KINETIC PHASE}

Using the initial conditions given by Eqs. (4.17)-(4.19), the evolution of the fluctuations can be solved in slightly different but, ultimately, equivalent ways. In the following, as a first step, the asymptotes for the evolution of the fluctuations will be discussed analytically in the vicinity of $t_{\mathrm{e}}$. The consistency of the analytical solutions with the results of the numerical integration is an important check to be done. Following recent techniques developed in a different framework [16], the solutions in the vicinity of $t_{\mathrm{e}}$ can be obtained without specifying the form of the potential for the field $\psi$. Later on, in order to perform the numerical integration, the case of massive potential will be mainly discussed.

\section{A. The initial stages around $t_{\mathrm{e}}$ for generic potential}

During this phase the field $\psi$ slowly rolls down its own potential $W(\psi)$, and the solution to Eq. (2.4) is given by Eq. (2.12). The same expansion in the gradients of the potential $W(\psi)$ can be used in order to get the approximate evolution of the fluctuations.

Equations (4.8) and (4.9) can then be approximately solved by keeping the leading terms in the derivatives of $W(\psi)$. As done in the case of the background evolution, an equation analogous to Eq. (2.12) can also be obtained for the canonical perturbation variable $v_{\psi}$. Equation (4.9) can then be expanded in gradients of the potential leading to the following approximate equation:

$$
\dot{v}_{\psi}=-\frac{1}{6 H} \frac{\partial^{2} W}{\partial \psi^{2}} v_{\psi},
$$

whose solution implies that $v_{\psi}$ is approximately constant. For instance, inserting into Eq. (5.1) the explicit expression for $W(\psi)$ given in Eq. (2.6), we get

$$
v_{\psi}(k, t) \simeq v_{\psi}\left(k, t_{\mathrm{e}}\right)\left[1-\frac{m^{2}}{4}\left(t^{2}-t_{\mathrm{e}}^{2}\right)\right], \quad t<t_{\mathrm{m}} .
$$

This expression has been verified numerically for various values of the physical parameters. Using Eq. (5.2), Eq. (4.8) can be solved in the same approximation. Note, in fact, that in Eq. (4.8) the term in square brackets is subleading with respect to the other terms for two separate reasons. First $V_{, \varphi \varphi}$ is negligible in its own right, given the smallness of $\lambda$ and recalling that $M / M_{\mathrm{P}}$ is $\mathcal{O}\left(10^{-13}\right)$. Second, since $a^{3} \dot{\varphi}^{2} / H$ is constant, its time derivative appearing in Eq. (4.8) is also negligible. The approximate evolution of $v_{\varphi}(k, t)$ is then given, at large scales, by

$$
\ddot{v}_{\varphi}+3 H \dot{v}_{\varphi}-\frac{3}{M_{\mathrm{P}}^{2} a^{3}} \frac{\partial}{\partial t}\left(\frac{a^{3}}{H} \dot{\varphi} \dot{\psi}\right) v_{\psi}=0,
$$

whose solution, using Eqs. (2.12) and (5.1), becomes

$$
v_{\varphi}(k, t) \simeq-\frac{3}{4 M_{\mathrm{P}}^{2}}\left(\frac{\dot{\varphi}}{H}\right) \frac{\partial W}{\partial \psi} v_{\psi}(k, t)\left[a^{6}(t)-a^{6}\left(t_{\mathrm{e}}\right)\right] .
$$


Given that during the kinetic phase $\dot{\varphi} \simeq H$, then $v_{\varphi}(k, t)$ $\propto a^{6}$. Again, in the case of Eq. (2.6), from Eq. (5.4) we obtain

$$
v_{\varphi}(k, t) \simeq-\frac{3 \sqrt{2}}{4}\left(\frac{\psi_{\mathrm{e}}}{M_{\mathrm{P}}}\right) m^{2}\left(t^{2}-t_{\mathrm{e}}^{2}\right) \chi_{\psi}\left(k, t_{\mathrm{e}}\right),
$$

where, according to Eq. (4.18), $v_{\psi}\left(k, t_{\mathrm{e}}\right)=\chi_{\psi}\left(k, t_{\mathrm{e}}\right)$ has been used. With the results of Eqs. (5.2) and (5.5), from Eqs. (4.10)-(4.12) we can also obtain the approximate form of the evolution of $\phi_{k}(t), \zeta_{k}(t)$, and $\chi_{\varphi}(k, t)$ :

$$
\begin{aligned}
\zeta_{k}(t) & \simeq \frac{3}{2 M_{\mathrm{P}}^{2}} \frac{\partial W}{\partial \psi} \chi_{\psi}\left(k, t_{\mathrm{e}}\right)\left[a^{6}(t)-a^{6}\left(t_{\mathrm{e}}\right)\right], \\
\phi_{k}(t) & \simeq-\frac{3}{10} \zeta_{k}(t), \\
\chi_{\varphi}(k, t) & \simeq-\frac{3}{10 M_{\mathrm{P}}^{2}}\left(\frac{\dot{\varphi}}{H}\right) \frac{\partial W}{\partial \psi} \chi_{\psi}\left[a^{6}(t)-a^{6}\left(t_{\mathrm{e}}\right)\right] .
\end{aligned}
$$

The same results derived in Eqs. (5.6) - (5.8) and based on the solution of Eqs. (4.8) and (4.9), with the initial conditions dictated by Eq. (4.18), can be obtained by integrating Eqs. (4.4)-(4.6). As a check of the consistency of the approach, it is in fact useful to insert Eqs. (5.7) and (5.8) back into Eqs. (4.3)-(4.6) and see that they are satisfied during the kinetic phase and prior to the oscillations of $\psi$. Furthermore, the evolution of $\zeta_{k}$, as obtained in Eq. (5.6), can be also obtained directly from Eq. (4.14). In fact, during the kinetic phase $\dot{\psi} \ll \dot{\varphi}$ and, from Eq. (4.16) evaluated in the kinetic limit:

$$
c_{s}^{2} \simeq 1-\frac{1}{18 M_{\mathrm{P}}^{2} H^{4}} \frac{\partial W}{\partial \psi} .
$$

Hence, from Eq. (4.15) we find

$$
\delta p_{\text {nad }}=-2 \frac{\partial W}{\partial \psi} \chi_{\psi}
$$

Inserting Eq. (5.10) into Eq. (4.14) and performing the integral, we get exactly Eq. (5.6). The analytic expressions derived so far allow a full control of the initial conditions of the system in the vicinity of $t_{\mathrm{e}}$.

\section{B. Evolution for $t_{\mathrm{e}}<t<t_{\mathrm{d}}$}

After the onset of the kinetic phase at $t_{\mathrm{e}}$, but before the dominance of $\psi$ at $t_{\mathrm{d}}$, the evolution of the system can be solved numerically for various sets of initial conditions; an example of this behavior is reported in Fig. 3 for the case of the potential given in Eq. (2.6).

It will now be shown that the numerical evolution can be very accurately reproduced analytically by direct integration of the evolution equation in a well defined approximation scheme. The evolution of the fluctuations will be analyzed first using Eqs. (4.8) and (4.9) and then using directly the
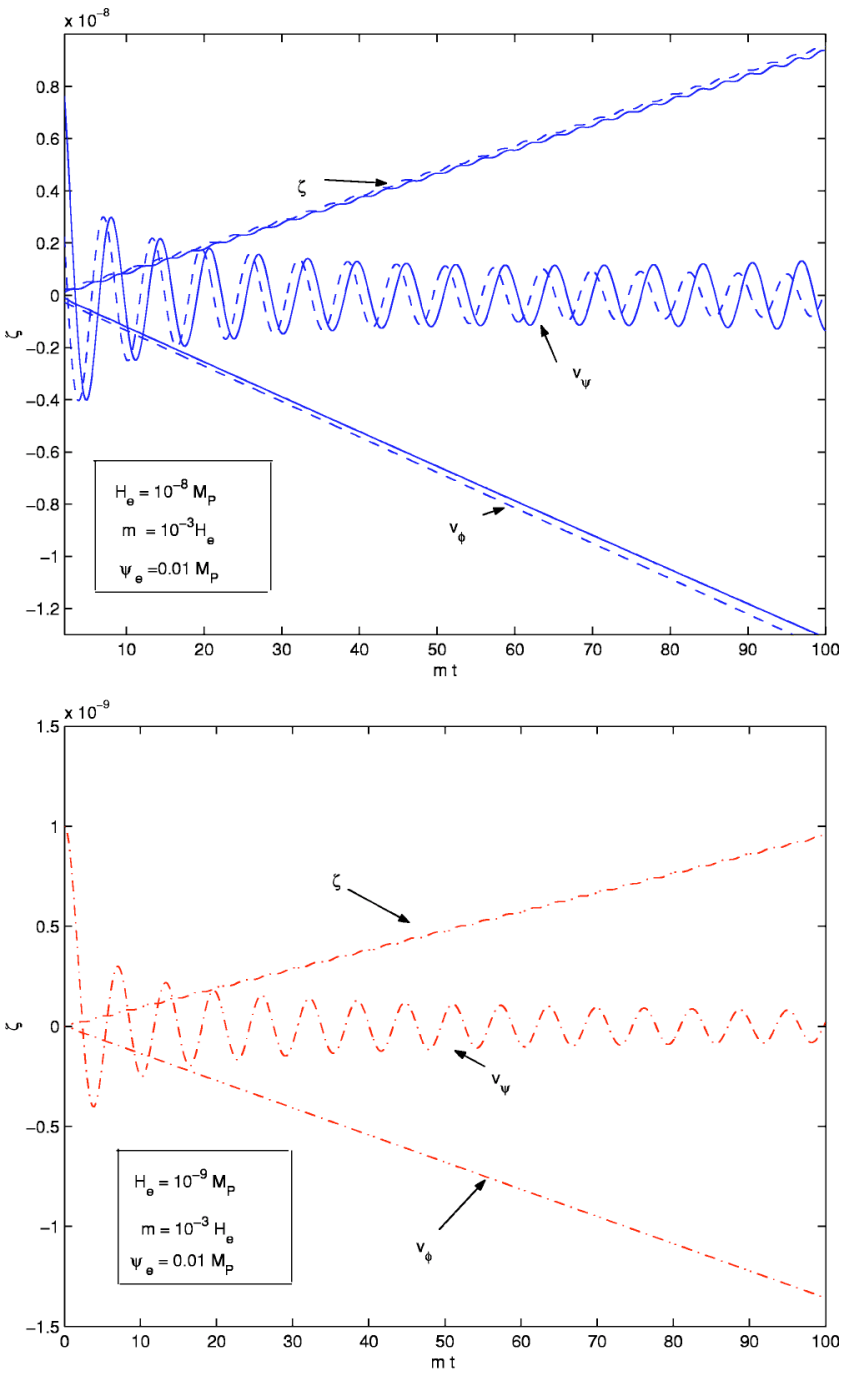

FIG. 3. The result of the numerical integration for the evolution of the fluctuations is illustrated for the case $M / M_{\mathrm{P}}=10^{-13}$ and for a set of fiducial parameters chosen within the shaded region of Fig. 2 . In the left plot the analytical results (dashed lines) obtained in Eqs. (5.14), (5.17) and (5.19) are compared with the numerical ones (full lines).

evolution equation for $\zeta$, i.e. Eq. (4.14).

In the case of the potential (2.6), Eqs. (4.8) and (4.9) can be written as

$$
\begin{gathered}
\frac{d}{d t}\left(a^{3} \dot{v}_{\varphi}\right)=-\frac{3 \sqrt{2}}{M_{\mathrm{P}}} m^{2} \psi v_{\psi}, \\
\ddot{v}_{\psi}+3 H \dot{v}_{\psi}+m^{2}\left[1+\frac{6}{H M_{\mathrm{P}}^{2}} \dot{\psi} \psi\right] v_{\psi} \\
+\frac{3 \sqrt{2}}{M_{\mathrm{P}}} m^{2} \psi v_{\varphi}=0,
\end{gathered}
$$

where Eqs. (2.11) and (2.4) have been used. Recalling now the exact solution for the evolution of Eq. (2.4), i.e. Eq. (2.14), it can be easily checked that, in Eq. (5.12): 


$$
\frac{6}{H M_{\mathrm{P}}^{2}} \dot{\psi} \psi \sim \frac{18 t}{M_{P}^{2}} \psi_{\mathrm{e}}^{2} m<1
$$

for $t<t_{\mathrm{d}} \sim m^{-1}\left(M_{\mathrm{P}} / \psi_{\mathrm{e}}\right)^{2}$. Neglecting the term containing $v_{\varphi}$ in Eq. (5.12), the solution for the evolution of $v_{\psi}$ is given by

$$
v_{\psi}(k, t)=\frac{v_{\psi}\left(k, t_{\mathrm{e}}\right)}{J_{0}\left(x_{\mathrm{e}}\right)} J_{0}(x),
$$

where $x=m t$ and $J_{0}(x)$ is the Bessel function of order 0 [30]. Equation (5.14) reproduces exactly the numerical solutions for $v_{\psi}$ reported, for a particular case, in Fig. 3. Since it is always true that $m t_{\mathrm{e}} \ll 1$ for the constraints displayed in Fig. 2, the interesting limits of Eq. (5.14) are for $m t \ll 1$ and $m t \gg 1$. In the limit $m t \ll 1$, Eq. (5.14) reproduces, as it should, the time dependence obtained in Eq. (5.2). For $m t$ $\gg 1$ we have [30], from Eq. (5.14):

$$
v_{\psi}(k, t)=v_{\psi}\left(k, t_{\mathrm{e}}\right) \sqrt{\frac{2}{\pi x}} \cos \left(x-\frac{\pi}{4}\right) .
$$

Note the similarity between Eqs. (5.14) and (2.14), which is a simple consequence of the quadratic form of the potential.

Inserting now Eq. (5.14) in Eq. (5.11), and integrating a first time between $t_{\mathrm{e}}$ and a generic time $t$ we get

$$
\begin{aligned}
\frac{d v_{\varphi}}{d x}= & -\frac{3 \sqrt{2}}{2 M_{\mathrm{P}}} \frac{\psi_{\mathrm{e}} v_{\psi}\left(k, t_{\mathrm{e}}\right)}{J_{0}\left(x_{\mathrm{e}}\right)}\left[x \left[J_{0}^{2}(x)\right.\right. \\
& \left.\left.+J_{1}^{2}(x)\right]-\frac{F\left(x_{\mathrm{e}}\right)}{x}\right],
\end{aligned}
$$

where $F\left(x_{\mathrm{e}}\right)=x_{\mathrm{e}}^{2}\left[J_{0}\left(x_{\mathrm{e}}\right)^{2}+J_{1}\left(x_{\mathrm{e}}\right)^{2}\right]$.

Direct integration of Eq. (5.16) implies that

$$
v_{\varphi}(k, x)=-\frac{3 \sqrt{2}}{4} \frac{\psi_{\mathrm{e}} v_{\psi}\left(k, t_{\mathrm{e}}\right)}{J_{0}\left(x_{\mathrm{e}}\right)^{2}}\left\{x^{2}\left[J_{0}^{2}(x)+2 J_{1}^{2}(x)-J_{0}(x) J_{2}(x)\right]-G\left(x_{\mathrm{e}}\right)-2 F\left(x_{\mathrm{e}}\right)\right\},
$$

where $\quad G\left(x_{\mathrm{e}}\right)=x_{\mathrm{e}}^{2}\left[J_{0}^{2}\left(x_{\mathrm{e}}\right)+2 J_{1}^{2}\left(x_{\mathrm{e}}\right)-J_{0}\left(x_{\mathrm{e}}\right) J_{2}\left(x_{\mathrm{e}}\right)\right] \quad$ and where, as usual, $x_{\mathrm{e}} \sim m / H_{\mathrm{e}}$. Taking the limit for large $x$ of Eq. (5.17) the following result can be obtained:

$$
\begin{aligned}
v_{\varphi}(k, t)= & -\frac{6 \sqrt{2}}{\pi} \psi_{\mathrm{e}} v_{\psi}\left(k, t_{\mathrm{e}}\right)(m t) \\
& +\frac{3 \sqrt{2}}{4} \psi_{\mathrm{e}} v_{\psi}\left(k, t_{\mathrm{e}}\right)\left(m t_{\mathrm{e}}\right)^{2} \ln \frac{t}{t_{\mathrm{e}}},
\end{aligned}
$$

showing off the linear growth of $v_{\varphi}$ with time.

Inserting now the solutions for $v_{\psi}$ and $v_{\varphi}$ given, respectively, in Eqs. (5.14) and (5.17) into Eq. (4.12), the evolution of $\zeta$ for $t<t_{\mathrm{d}}$ turns out to be, for $m t>1$,

$$
\zeta(k, t) \simeq \frac{6}{\pi}\left(\frac{\psi_{\mathrm{e}}}{M_{\mathrm{P}}}\right)\left[\frac{v_{\psi}\left(k, t_{\mathrm{e}}\right)}{M_{\mathrm{P}}}\right](m t) .
$$

Note that, for $t=t_{\mathrm{d}}$, Eq. (5.19) gives

$$
\zeta\left(k, t_{\mathrm{d}}\right)=\frac{2}{5 \pi}\left(\frac{v_{\psi}\left(k, t_{\mathrm{e}}\right)}{\psi_{\mathrm{e}}}\right) .
$$

Using the result of Eq. (5.19) into Eq. (4.13) the evolution for the large-scale modes of the metric fluctuations can be obtained:

$$
\phi(k, t) \simeq-\frac{18}{7 \pi}\left(\frac{\psi_{\mathrm{e}}}{M_{\mathrm{P}}}\right)\left[\frac{v_{\psi}\left(k, t_{\mathrm{e}}\right)}{M_{\mathrm{P}}}\right](m t) .
$$

Again we verified that the same results can be obtained directly by integrating the Hamiltonian constraint (4.4).
The time has come to compare the accuracy of the analytical expressions derived in Eqs. (5.14), (5.17) and (5.19). These expressions have been plotted in Fig. 3 (left plot) with the dashed lines. For the same set of parameters (and with the full line) the outcome of the numerical integration has been reported. In the right plot the results for a different set of parameters are displayed. The analytical expressions match rather accurately the numerical results. Notice that the small wiggles in the evolution of $\zeta(k, t)$, modulating the linear growth, are due to the fact that we decided to plot the full expression of $\zeta(k, t)$, which contains Bessel functions, and not only its asymptotic limit for $m t>1$. The same evolution for $\zeta(k, t)$ derived in this section can be directly inferred from Eq. (4.14), recalling the approximate form of $\delta p_{\text {nad }}$. This calculation is reported in the Appendix.

It is now appropriate to compare the situation of low-scale quintessential inflation with the situation occurring in the more conventional case of curvaton models where the inflationary phase is suddenly followed by the radiationdominated phase. In this case the $\zeta(k, t)$ variable grows as $\sqrt{t}$ (i.e. linearly in conformal time) before the curvaton becomes dominant. Here we found that the growth is linear in cosmic time. In spite of this difference, the final amplitude of $\zeta(k, t)$ is given approximately by $\chi_{\psi}\left(k, t_{\mathrm{d}}\right) / \psi_{\mathrm{e}}$. In fact, in the case of a quadratic potential evolving in a radiation-dominated environment,

$$
\dot{\zeta} \sim\left(\frac{\psi_{\mathrm{e}}}{M_{\mathrm{P}}}\right)\left[\frac{\chi_{\psi}\left(k, t_{\mathrm{e}}\right)}{M_{\mathrm{P}}}\right] H a(t),
$$

where we now have $a(t) \sim \sqrt{t}$. Integrating once the previous formula we get $\zeta(k, t) \sim \sqrt{t}$. If the evolution occurs during 
radiation, the curvaton will become dominant at a typical curvature scale $H_{\mathrm{d}} \sim m\left(\psi_{\mathrm{e}} / M_{\mathrm{P}}\right)^{4}$ [16]. Using this result, the amplitude of $\zeta\left(k, t_{\mathrm{d}}\right)$ is given, as previously anticipated, by $\chi_{\psi}\left(k, t_{\mathrm{d}}\right) / \psi_{\mathrm{e}}$.

\section{DOMINANCE OF $\psi$}

Since $\psi$ starts dominating the background at $t_{\mathrm{d}}$, for $t$ $>t_{\mathrm{d}}$ the evolution of the system is described by the following set of equations:

$$
\begin{gathered}
H^{2} M_{\mathrm{P}}^{2}=\left[\frac{\dot{\psi}^{2}}{2}+m^{2} \psi^{2}\right], \\
\dot{H} M_{\mathrm{P}}^{2}=-\frac{3}{2} \dot{\psi}^{2}, \\
\ddot{\psi}+3 H \dot{\psi}+m^{2} \psi=0 .
\end{gathered}
$$

For large times Eqs. (6.1)-(6.3) lead to an effectively matterdominated phase where the oscillations of $\psi$,

$$
\psi(t) \simeq \psi\left(t_{\mathrm{d}}\right) \frac{\cos m t}{\left(H_{\mathrm{d}} t\right)^{2}}
$$

induce oscillations in the Hubble parameter and in the scale factor, which increases, on average, as $t^{2 / 3}$.

It is not difficult to see that, under these conditions, the evolution of $v_{\psi}$ is dominated by a constant mode. In Eq. (4.9) the term containing $v_{\varphi}$ is always suppressed for large times since, from Eq. (3.4)

$$
\dot{\varphi} \simeq \frac{\sqrt{2}}{3} M_{\mathrm{P}} \frac{t_{\mathrm{d}}}{t^{2}}
$$

and the quintessence field goes very rapidly to a constant. Thus, in this regime, the evolution of $v_{\psi}$ is given by

$$
v_{\psi}(k, t)=v_{\psi}\left(k, t_{\mathrm{d}}\right) \frac{\dot{\psi}}{H} \text {. }
$$

Equation (4.8) allows us to deduce that

$$
v_{\varphi}(k, t) \sim t^{-3}
$$

Inserting now Eqs. (6.4)-(6.7) into Eq. (4.12) we find that, for $t>t_{\mathrm{d}}, \zeta$ is frozen to its constant value. Therefore, right before the decay of $\psi$, the metric fluctuation will be given by

$$
\phi\left(k, t_{\mathrm{r}}\right) \sim \frac{\chi_{\psi}\left(k, t_{\mathrm{e}}\right)}{\psi_{\mathrm{e}}} .
$$

After $t_{\mathrm{r}}$ the field $\psi$ decays and the evolution of the generated adiabatic mode becomes standard, namely we have the fluctuations of the quintessence field evolving in a radiation-dominated environment together with the constant mode of $\zeta$. The evolution equations for the fluctuations of the quintessence field will then obey, at large scales,

$$
\ddot{\chi}_{\varphi}+3 \dot{H} \dot{\chi}_{\varphi}+\frac{\partial^{2} V}{\partial \varphi^{2}} \chi_{\varphi}=4 \dot{\varphi} \dot{\phi}-2 \frac{\partial V}{\partial \varphi} \phi
$$

The other equations are the standard ones, namely

$$
\begin{gathered}
-3 H(H \phi+\dot{\phi}) \\
=\frac{3}{2 M_{\mathrm{P}}^{2}} \rho_{\mathrm{r}} \delta_{\mathrm{r}}+\frac{3}{2 M_{\mathrm{P}}^{2}}\left[-\phi \dot{\varphi}^{2}+\dot{\varphi} \dot{\chi}_{\varphi}+\frac{\partial V}{\partial \varphi} \chi_{\varphi}\right] \\
\ddot{\phi}+4 H \dot{\phi}+\left(3 H^{2}+2 \dot{H}\right) \phi \\
=\frac{1}{2 M_{\mathrm{P}}^{2}} \rho_{\mathrm{r}} \delta_{\mathrm{r}}-\frac{3}{2 M_{\mathrm{P}}^{2}}\left[\dot{\varphi}^{2} \phi-\dot{\chi}_{\varphi}+\frac{\partial V}{\partial \varphi} \chi_{\varphi}\right] \\
H \phi+\dot{\phi}=\frac{3}{2 M_{\mathrm{P}}^{2}}\left[\dot{\varphi} \chi_{\varphi}+\frac{4}{3} \rho_{\mathrm{r}} u_{\mathrm{r}}\right] \\
\dot{\delta}_{\mathrm{r}}-4 \dot{\phi}=0 \\
\dot{u}_{\mathrm{r}}-\frac{1}{4} \delta_{\mathrm{r}}-\phi=0
\end{gathered}
$$

where $\delta_{\mathrm{r}}=\delta \rho_{\mathrm{r}} / \rho_{\mathrm{r}}$ and $u_{\mathrm{r}}$ is the velocity potential.

As discussed in the context of the quintessential evolution of the background, the quintessence field, in the present model, does not lead to a tracking behavior, as also noticed in [9]. Thus, the evolution of the fluctuations during the radiation-dominated stage of expansion will effectively be the one implied by a standard cosmological term. In fact we can recall, from Eq. (3.5), that $\varphi$ approaches a constant value as $t^{-1 / 2}$ while the potential is constant. Then, from Eq. (6.9) it can be deduced that also $\chi_{\varphi} \sim t^{-1 / 2}$ at large scales. As a consequence, combining Eqs. (6.10) and (6.11) we have, at large scales,

$$
\ddot{\phi}+5 H \dot{\phi}+2\left(\dot{H}+2 H^{2}\right) \phi=0,
$$

leading to the usual constant mode which was present prior to matter-radiation equality, a known feature of these types of models [31].

\section{CONCLUDING REMARKS}

In the present paper, low-scale quintessential inflationary models have been analyzed. The setup of the model is closely related to the one proposed by Peebles and Vilenkin. Even if the curvature scale at the end of inflation is much smaller than $10^{-6} M_{\mathrm{P}}$, curvature perturbations of correct amplitude can be generated. In these models, the quintessence and the inflaton field are identified. The consistency of the background evolution imposes a number of constraints on the various parameters. Assuming a specific form of the 
inflaton/quintessence potential and a massive curvaton, the evolution of the fluctuations has been followed through the different stages of the model. Even if, for some region of the parameter space of the model, non-Gaussian (adiabatic) fluctuations can be generated, the case of the Gaussian adiabatic mode has been discussed. The curvaton evolution occurring in low-scale quintessential inflation is different from the one possibly obtained in the case when the radiation-dominated evolution follows immediately inflation. In low-scale quintessential inflation, prior to curvaton dominance, curvature perturbations grow linearly in cosmic time. On the contrary, if inflation is immediately followed by a radiation-dominated stage, then curvature fluctuations grow as $\sqrt{t}$.

In spite of the fact that low-scale quintessential inflationary models share essential analogies with the prediction of ordinary quintessential inflation there are also relevant differences between them. In ordinary quintessential inflationary models, the reheating is mainly gravitational. In the present case the reheating is triggered by the curvaton decay. In ordinary quintessential inflationary models a large background of gravitational waves should be expected in the $\mathrm{GHz}$ region $[32,33]$. In the case of low-scale quintessential inflation, gravitational waves are negligible over all the frequencies of the spectrum and, in particular, at over the typical frequency range of wide-band interferometers $[34,35]$.

\section{ACKNOWLEDGMENTS}

The author wishes to thank, V. Bozza, M. Gasperini, and G. Veneziano for useful discussions.

\section{APPENDIX: EVOLUTION OF $\zeta$ DURING THE KINETIC PHASE}

In this appendix the analytical solution of Eq. (4.14) will be obtained for $t_{\mathrm{e}}<t<t_{\mathrm{d}}$. During the kinetic phase, $\dot{\varphi}^{2}$ $\gg \dot{\psi}^{2}$. Furthermore, as discussed in the paper

$$
\delta p_{\mathrm{nad}} \simeq-2 \frac{\partial W}{\partial \psi} \chi_{\psi}=-2 m^{2} \psi \chi_{\psi}
$$

Thus, from Eq. (4.14), we obtain that

$$
\frac{d \zeta}{d t}=\frac{2 H}{\dot{\varphi}^{2}} m^{2} \psi \chi_{\psi}
$$

For $t_{\mathrm{e}}<t<t_{\mathrm{d}}$, the approximate evolution of $\psi$ and $\chi_{\psi}$ is given by

$$
\begin{gathered}
\psi(t)=\psi_{\mathrm{e}} \frac{\sqrt{t}}{a^{3 / 2}} \frac{J_{0}(m t)}{J_{0}\left(m t_{\mathrm{e}}\right)}, \\
\chi_{\psi}(t)=\chi_{\psi}\left(k, t_{\mathrm{e}}\right) \frac{\sqrt{t}}{a^{3 / 2}} \frac{J_{0}(m t)}{J_{0}\left(m t_{\mathrm{e}}\right)} .
\end{gathered}
$$

Inserting these solutions into Eq. (A2) we get

$$
\begin{aligned}
\zeta(k, t)= & \frac{3}{2}\left(\frac{\psi_{\mathrm{e}}}{M_{\mathrm{P}}}\right)\left(\frac{\chi_{\psi}\left(k, t_{\mathrm{e}}\right)}{M_{\mathrm{P}}}\right) \\
& \times\left\{(m t)^{2}\left[J_{0}^{2}(m t)+J_{1}^{2}(m t)\right]-\left(m t_{\mathrm{e}}\right)^{2}\right\},
\end{aligned}
$$

where we used the fact that $m t_{\mathrm{e}} \ll 1$ because of the constraint stemming from Eq. (2.20). Taking the limit of Eq. (A4) for $m t \gg 1$ we obtain

$$
\zeta(k, t)=\frac{6}{\pi}\left(\frac{\psi_{\mathrm{e}}}{M_{\mathrm{P}}}\right)\left(\frac{\chi_{\psi}\left(k, t_{\mathrm{e}}\right)}{M_{\mathrm{P}}}\right) m t,
$$

which is the same as Eq. (5.19) since at $t_{\mathrm{e}}$, according to Eq. (4.18), $\chi_{\psi}\left(k, t_{\mathrm{e}}\right) \equiv v_{\psi}\left(k, t_{\mathrm{e}}\right)$.
[1] A.G. Riess et al., Astron. J. 116, 1009 (1998).

[2] S. Perlmutter et al., Astrophys. J. 517, 565 (1999).

[3] P.J.E. Peebles and B. Ratra, Astrophys. J. Lett. 325, L17 (1988).

[4] B. Ratra and P.J.E. Peebles, Phys. Rev. D 37, 3406 (1988).

[5] R.R. Caldwell, R. Dave, and P.J. Steinhardt, Phys. Rev. Lett. 80, 1582 (1998).

[6] L. Wang and P.J. Steinhardt, Astrophys. J. 508, 483 (1998).

[7] I. Zlatev, L. Wang, and P.J. Steinhardt, Phys. Rev. Lett. 82, 896 (1999).

[8] S.M. Carroll, Phys. Rev. Lett. 81, 3067 (1998).

[9] P.J.E. Peebles and A. Vilenkin, Phys. Rev. D 59, 063505 (1999).

[10] P.J.E. Peebles and A. Vilenkin, Phys. Rev. D 60, 103506 (1999).

[11] P.J.E. Peebles and B. Ratra, “The Cosmological Constant and Dark Energy," astro-ph/0207347.

[12] B. Spokoiny, Phys. Lett. B 315, 40 (1993).

[13] R.A. Frewin and J.E. Lidsey, Int. J. Mod. Phys. D 2, 323 (1993).
[14] K. Enqvist and M.S. Sloth, Nucl. Phys. B626, 395 (2002).

[15] D.H. Lyth and D. Wands, Phys. Lett. B 524, 5 (2002).

[16] V. Bozza et al., Phys. Lett. B 543, 14 (2002); Phys. Rev. D 67, 063514 (2003).

[17] M.S. Sloth, Nucl. Phys. B656, 239 (2003).

[18] T. Moroi and T. Takahashi, Phys. Lett. B 522, 15 (2001).

[19] M. Bastero-Gil, V. Di Clemente, and S.F. King, Phys. Rev. D 67, 103516 (2003).

[20] C. Gordon and A. Lewis, following article, Phys. Rev. D 67, 123513 (2003).

[21] S. Mollerach, Phys. Rev. D 42, 313 (1990).

[22] N. Bartolo and A. Liddle, Phys. Rev. D 65, 121301(R) (2002).

[23] V.F. Mukhanov, H.A. Feldman, and R.H. Brandenberger, Phys. Rep. 215, 203 (1992).

[24] J.M. Bardeen, Phys. Rev. D 22, 1882 (1980).

[25] J. Hwang, Phys. Rev. D 48, 3544 (1993); Class. Quantum Grav. 11, 2305 (1994).

[26] J. Hwang, Phys. Rev. D 53, 762 (1996); gr-qc/9608018.

[27] J. Hwang, Astrophys. J. 375, 443 (1991).

[28] H. Kodama and M. Sasaki, Prog. Theor. Phys. Suppl. 78, 
1 (1984).

[29] A. Taruya and Y. Nambu, Phys. Lett. B 428, 37 (1998).

[30] A. Erdelyi, W. Magnus, F. Obehettinger, and F. R. Tricomi, Higher Transcendental Functions (McGraw-Hill, New York, 1953).

[31] P. Viana and A. Liddle, Phys. Rev. D 57, 674 (1998).
[32] M. Giovannini, Class. Quantum Grav. 16, 2905 (1999).

[33] M. Giovannini, Phys. Rev. D 60, 123511 (1999).

[34] D. Babusci and M. Giovannini, Phys. Rev. D 60, 083511 (1999).

[35] D. Babusci and M. Giovannini, Class. Quantum Grav. 17, 2621 (2000). 